\title{
Comparación de destiladores solares para la obtención de agua dulce en pozos de agua salobre en la Comunidad Campesina San Juan Bautista de Catacaos - Piura
}

Comparison of solar distillers for the obtaining of fresh water in wells of brackish water in the Peasant Community San Juan Bautista of Catacaos Piura

Roberto Chiroque LujáN ${ }^{1}$

\section{RESUMEN}

Dada la necesidad de obtener agua dulce para consumo humano en beneficio de las asociaciones ganaderas ubicadas dentro de los linderos del desierto de Sechura y de la Comunidad Campesina San Juan Bautista de Catacaos, en Piura, se hizo un estudio científico experimental que tuvo como objetivo principal comparar diferentes modelos de destiladores solares, durante al menos 3 meses, usando agua salobre del pozo artesanal de ganaderos en el sector Tabanco. Para determinar el diseño de destilador solar productor de agua dulce más adecuado, desde el punto de vista, ambiental y económico la investigación aplicó el modelo estadístico Bifactorial de Bloques al Azar e hizo el análisis de varianza que se complementó con la prueba Tukey; obteniéndose como resultado (con un intervalo de confianza del $95 \%$ ) que el destilador solar de dos vertientes es el diseño más adecuado para las condiciones ambientales del desierto norteño permitiendo una producción de 1,509 a 1,767 litros de agua por día/ $\mathrm{m}^{2}$ de espejo de agua, para el periodo de evaluación de los meses de noviembre a diciembre; es también el más económico, presenta un menor costo de 34,1 soles en un horizonte de 10 años para tener 20 litros de agua destilada por día.

Palabras clave: comparación; modelos de destiladores; destiladores solares; obtención de agua destilada para consumo humano.

\footnotetext{
1 Universidad Nacional de Piura. Piura, Perú.

(C) Los autores. Este artículo es publicado por la Revista Aporte Santiaguino de la Universidad Nacional Santiago Antúnez de Mayolo. Este es un artículo de acceso abierto, distribuido bajo los términos de la Licencia Creative Commons Atribución-NoComercial-CompartirIgual 4.0 Internacional. (http://creativecommons.org/licenses/ by-nc-sa/4.0/), que permite el uso no comercial, distribución y reproducción en cualquier medio, siempre que la obra original sea debidamente citada.
} 


\begin{abstract}
Existing the need to obtain fresh water for human consumption for the benefit of livestock associations located within the boundaries of the Sechura desert and the San Juan Bautista de Catacaos Peasant Community, in Piura, an experimental scientific study was carried out with the main objective compare different models of solar distillers, for at least 3 months, using brackish water from the artisanal well of farmers in the Tabanco sector. (with a 95\% confidence interval) In order to determine the design of the best suitable freshwater solar distiller, from the point of view, environmental and economic, the research applied the Bifactorial statistical model of Random Blocks and did the analysis of variance that was complemented with the Tukey test; obtaining as a result that the solar distiller of two slopes is the most suitable design for the environmental conditions of the northern desert allowing a production of 1,509 to 1,767 liters of water per day $/ \mathrm{m}^{2}$ of water mirror, for the evaluation period of the months of November to December, it is also the most economical, with a lower cost of 34.1 soles over a 10 -year horizon to have 20 liters of distilled water per day.
\end{abstract}

Keywords: comparison, distillers models, solar distillers, obtaining distilled water for human consumption.

\title{
INTRODUCCIÓN
}

La destilación de agua con energía solar puede ser una solución económica a los problemas de escasez de agua dulce en pequeñas comunidades como lo afirma el informe del United Nations Department of Economic and Social Affairs (1970). En Piura, el poblador rural del bosque seco, especialmente el ganadero de la comunidad campesina San Juan Bautista de Catacaos, vive afrontando cada día el problema de escasés del agua, factor que los limita en su desarrollo económico e integral. Con la finalidad de encontrar alternativas que ayuden a solucionar este problema, se propone la utilización de destiladores solares que transforman el agua salada y amarga de las norias del desierto en agua dulce apta para el consumo humano y ganadero. A fin de hacer viable esta posibilidad se hace un estudio comparativo de diferentes modelos de destiladores solares en diferentes meses del año que nos permite conocer, a partir de un indicador de producción económico y válido, la oportunidad de introducir estos sistemas en estos grupos sociales de esta región norteña. Los destiladores solares son recomendados para la transformación de agua salada, McCluney (1984) y representan una de las mejores alternativas por la calidad de agua obtenida a bajo costo.

A nivel nacional, son escasos los trabajos desarrollados sobre evaluación de modelos de destiladores solares para determinar volúmenes de agua destilada de producción; en este contexto, el profesor-investigador Erich (2015), docente de la Facultad de Inge- 
niería Industrial de la Universidad de Lima demuestra que el destilador más sencillo y barato, llamado destilador de bandeja escalonada con paredes reflectoras, es un $40 \%$ más eficiente que otros en la producción de agua desalada. Asimismo, confirma que la calidad del agua obtenida cumple con los estándares nacionales para su consumo, esto lo señala en el XXII Simposio Peruano de Energía Solar y del Ambiente (XXII SPES). También a nivel de las investigaciones en América, Fonseca et al. (2009) presenta un prototipo de destilador solar de bandeja, construido en fibra de vidrio con la finalidad de obtener agua destilada para ser empleada en baterías. Estudia tener identificadas o localizadas las mayores pérdidas de líquido producido y mejorar posteriormente su diseño. La productividad del equipo alcanza valores de $350 \mathrm{ml}$ al día, lo cual representa 2,2 L/m2 día, similar al de otros prototipos desarrollados en el CIES en Santiago de Cuba y en correspondencia a lo que debe esperarse de acuerdo a las condiciones ambientales existentes (nivel de radiación, temperatura ambiente, velocidad del viento).

Vemos también críticas y aportes como las de Ros (2011) quien señala:

La energía solar es el método ideal para producir agua en zonas áridas y muy aisladas del resto de poblaciones. A pesar de tener un coste energético nulo y escasa inversión necesaria, su baja rentabilidad reside en su escasa producción por metro cuadrado de colector al destilarse tan sólo unos litros al día en el caso de condiciones climatológicas favorables.

Por lo tanto, no se han desarrollado a gran escala en lugares con un consumo elevado de agua dulce y sólo es posible pensar en usar estas instalaciones en sitios totalmente aislados y faltos de suministro de electricidad y agua.

Así pues, con el estudio de diferentes modelos de destiladores solares durante los meses de octubre a diciembre en el año 2017, y con el objeto de determinar indicadores económicos y de producción de agua destilada acorde a la realidad y necesidad de este sector del bosque seco del Desierto de Sechura, es que se decide realizar la presente investigación aplicándola en la Comunidad Campesina San Juan Bautista de Catacaos del Departamento de Piura.

Esta investigación se clasifica como un experimento cuyos resultados son sometidos a evaluaciones estadísticas. Así mismo, se realiza el procesamiento de los datos, haciendo el uso del diseño de investigación Experimento Bifactorial de Bloque al Azar. Se usa el método Tukey para realizar la comparación de los resultados.

\section{MATERIALES Y MÉTODOS}

Se trata de una investigación experimental cuyo diseño consiste en un experimento Bifactorial de Bloques al azar, usando el método Fischer y Tukey para realizar las com- 
paraciones de dos a dos. La población muestral fueron 104 latas de 20 litros de agua salobre de la noria o pozo artesanal zona de ganaderos sector Tabanco, en el desierto de sechura, en la comunidad campesina San Juan Bautista de Catacaos; la muestra o unidad de análisis fueron 52 latas de 20 litros de agua del pozo artesanal seleccionado. La técnica para la recolección de la información fue por observación estructurada o sistemática, como se demuestra en la siguiente tabla:

Tabla 1. Plan de muestreo y comparación de variables con fecha de evaluación real

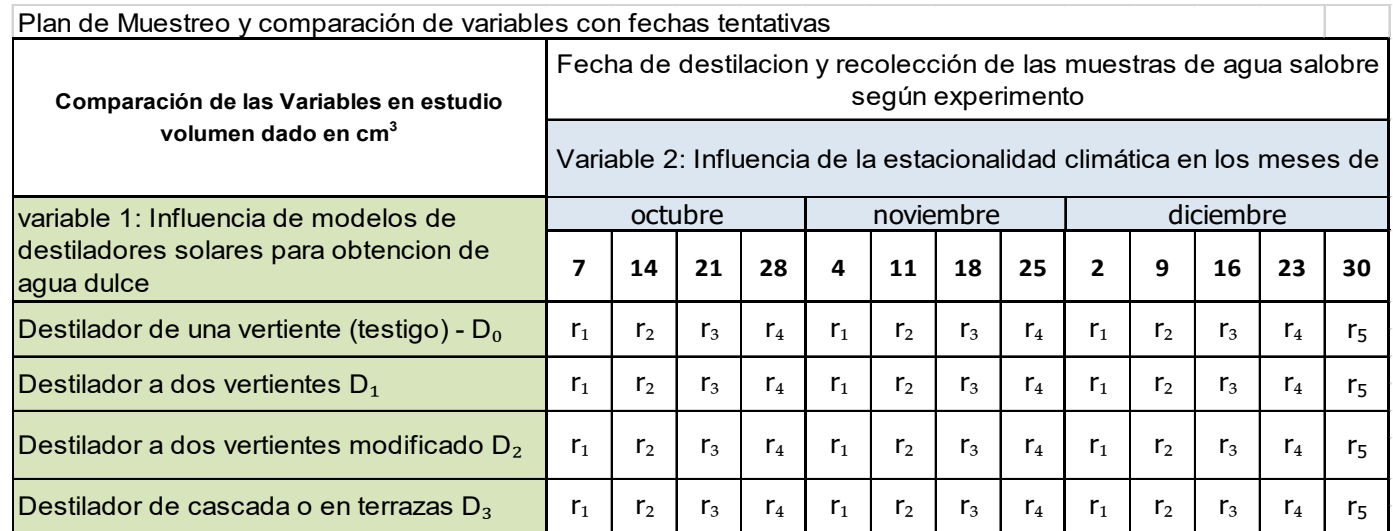

Los promedios obtenidos en cada tratamiento se compararon según el método Tukey y después de un ANOVA respectivo.

\section{RESULTADOS}

Los resultados del análisis de varianza indican que existen diferencias significativas (Sig. $<0,05)$ en el promedio de agua destilada obtenida en los meses de octubre, noviembre y diciembre; también se encontró diferencias significativas en el promedio de agua destilada obtenida con los diferentes destiladores.

El coeficiente de variación de 23,9\% indica que la variabilidad de los datos es relativamente baja, lo que les da confiabilidad a los resultados.

Asimismo, los mayores resultados de volumen de agua destilada obtenida por $\mathrm{m}^{2} \mathrm{de}$ espejo de agua tratada por día lo ha logrado el destilador solar de dos vertientes produciendo 1509 a 1763 en $\mathrm{cm}^{3} / \mathrm{m}^{2} /$ día de agua destilada en un intervalo de confianza del 95\% estadísticamente, como se puede apreciar en la tabla 2. 
Tabla 2. Cantidad en $\mathrm{cm}^{3} / \mathrm{m}^{2} /$ día promedio de agua obtenida con los destiladores y en los diferentes meses

\begin{tabular}{|c|c|c|c|c|c|}
\hline \multirow{2}{*}{ Destiladores } & \multicolumn{4}{|c|}{ MESES } & \multirow{2}{*}{$\begin{array}{c}\text { Intervalo de } \\
\text { confianza } \\
\text { del } 95 \%\end{array}$} \\
\hline & Octubre & Noviembre & Diciembre & Total & \\
\hline $\begin{array}{l}\text { Destilador solar de una } \\
\text { vertiente (Testigo) }\end{array}$ & $732 \mathrm{a}$ & $960 \mathrm{ab}$ & $1042 \mathrm{ab}$ & $921 \mathrm{a}$ & 784,1038 \\
\hline $\begin{array}{l}\text { Destilador solar de dos } \\
\text { vertientes }\end{array}$ & $1279 \mathrm{bc}$ & $1806 \mathrm{~cd}$ & $1823 \mathrm{~d}$ & $1651 \mathrm{~b}$ & 1509,1763 \\
\hline $\begin{array}{l}\text { Destilador solar de dos } \\
\text { vertientes modificado }\end{array}$ & $1070 \mathrm{ab}$ & $1465 \mathrm{bcd}$ & $1729 \mathrm{~cd}$ & $1445 \mathrm{~b}$ & 1294,1549 \\
\hline $\begin{array}{l}\text { Destilador escalonado } \\
\text { o en terrazas }\end{array}$ & $1094 \mathrm{ab}$ & $1691 \mathrm{~cd}$ & $1738 \mathrm{~cd}$ & $1525 \mathrm{~b}$ & 1380,1635 \\
\hline Total & $1044 \mathrm{a}$ & $1481 \mathrm{~b}$ & $1583 \mathrm{~b}$ & 1386 & \\
\hline Intervalo de confianza & 930 & 1367 & 1481 & & \\
\hline del $95 \%$ & 1158 & 1595 & 1685 & & \\
\hline
\end{tabular}

Luego de encontrar diferencias significativas en los promedios de agua destilada obtenidos en los diferentes meses y destiladores, se procede a comparar los pares de promedios usando la prueba Tukey.

Los resultados muestran que el mayor volumen promedio de agua destilada producida en los cuatro modelos de destiladores, se obtuvo en el mes de noviembre y diciembre, con un promedio de 1481 y $1583, \mathrm{cc} / \mathrm{m}^{2} /$ día respectivamente; en tanto que en el mes octubre el promedio fue de $1044 \mathrm{cc} / \mathrm{m}^{2} /$ día. (figura 01)

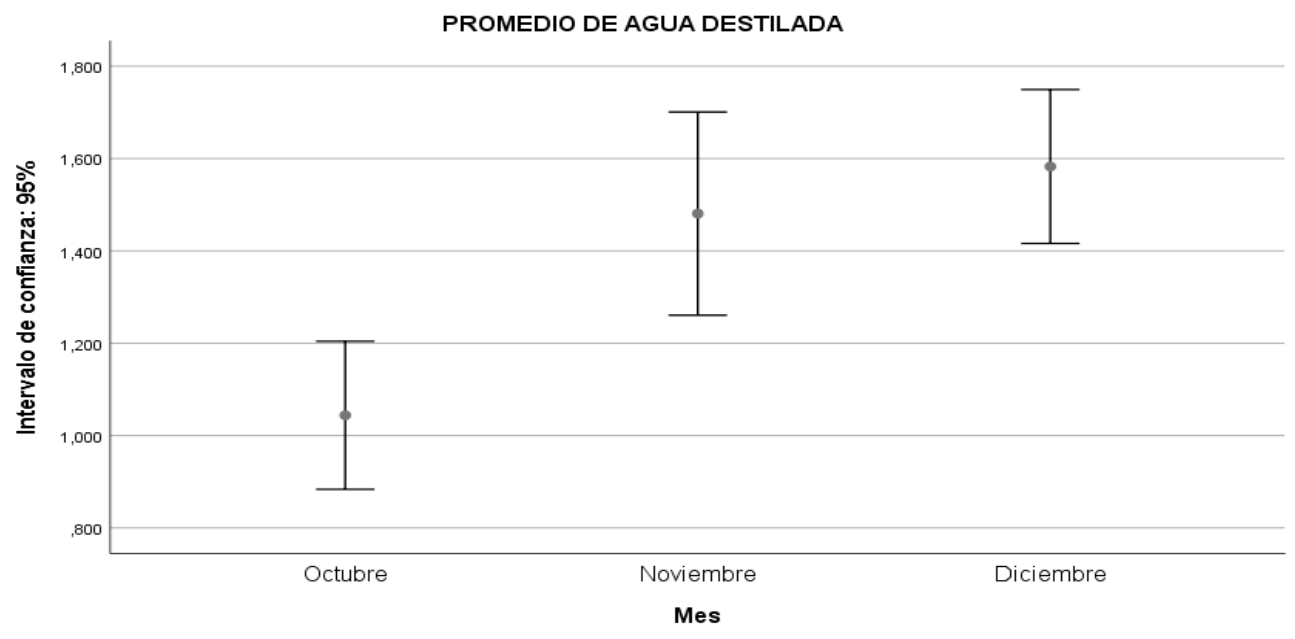

Figura 1. Promedio de volumen de agua destilada obtenida por mes 
En el caso de los destiladores, los mayores volúmenes de agua se logran con el destilador solar de dos vertientes, destilador solar a dos vertientes modificado y destilador solar de cascada, con promedios de 1651, 1445 y $1525 \mathrm{cc} / \mathrm{m}^{2} /$ día respectivamente.

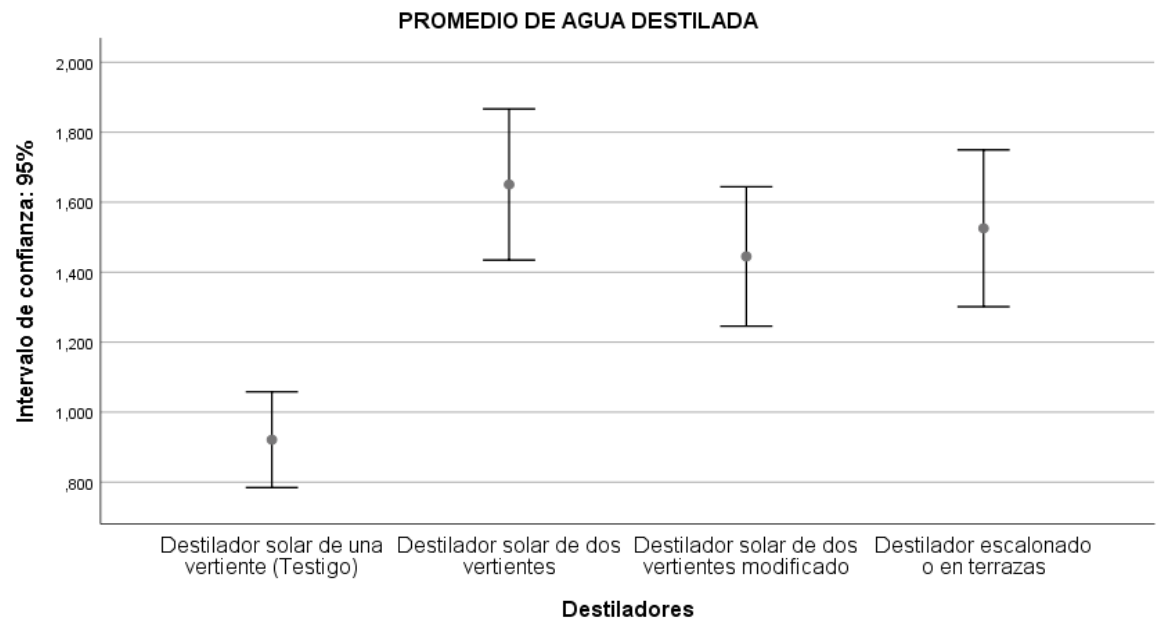

Figura 2. Volumen promedio de agua destilada según tipo de destilador $\mathrm{cc} / \mathrm{m}^{2} /$ día

Al evaluar las interacciones entre los factores climatológicos y el volumen de agua, los resultados indican que los mayores promedios se logran en el mes de diciembre y noviembre usando el destilador modificado de dos vertientes, con promedios de 1823 y $1806 \mathrm{cc} / \mathrm{m}^{2} /$ día respectivamente; también se logra promedios similares, pero ligeramente inferiores, en el mes de diciembre con destilador de cascada o en terrazas y el destilador solar de dos vertientes modificado, cuyos promedios son de 1729 y $1738 \mathrm{cc} / \mathrm{m}^{2} /$ día. En el mes de noviembre también se logró volúmenes similares, con destilador solar a dos vertientes modificado y con destilador escalonado o en terrazas, con volúmenes de agua de 1465 y $1691 \mathrm{cc} / \mathrm{m}^{2} /$ día respectivamente, como se aprecia gráficamente en la figura 3. 


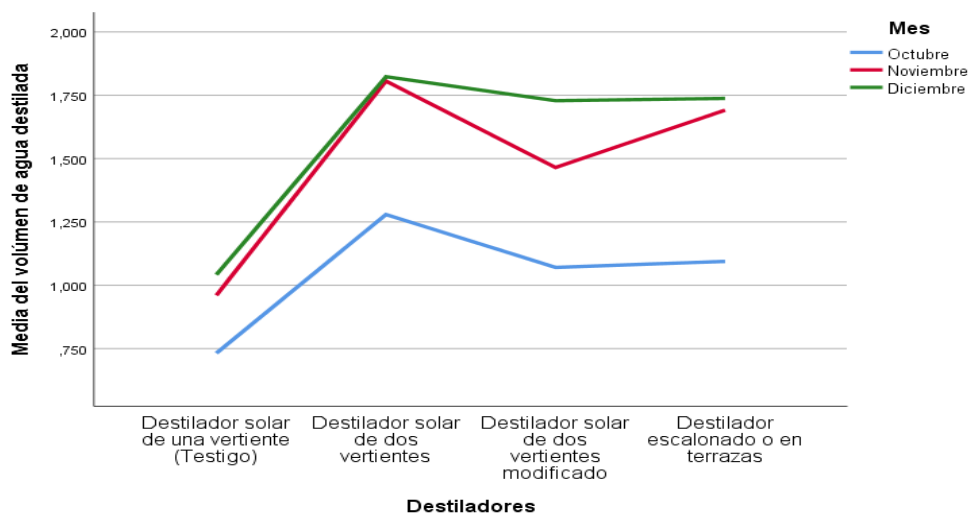

Figura 3. Volumen promedio de agua destilada por mes y tipo de destilador

Según la tabla 3, los resultados del análisis de correlación indican que el volumen de agua destilada obtenida con un destilador de una vertiente o el obtenido con un destilador solar de dos vertientes modificado, se relaciona en forma significativa $($ Sig. $<0,05)$ y directa con la temperatura media; el primer tipo de destilador también se relaciona significativamente con la temperatura mínima, mientras que el segundo evidencia este tipo de relación tanto con la temperatura mínima como con la temperatura máxima. Las correlaciones positivas implican que en la medida que dicha temperatura se incrementa, el volumen de agua destilada, obtenida con los destiladores mencionados también se incrementa.

Tabla 3. Relación entre los factores climatológicos y el volumen de agua obtenido con los destiladores

\begin{tabular}{|c|c|c|c|c|c|}
\hline $\begin{array}{c}\text { Factores } \\
\text { dimatológicos }\end{array}$ & Pearson & $\begin{array}{c}\text { Destilador } \\
\text { solar de una } \\
\text { vertiente }\end{array}$ & $\begin{array}{l}\text { Destilador } \\
\text { solar dedos } \\
\text { vertientes }\end{array}$ & $\begin{array}{l}\text { Destilador } \\
\text { solar de dos } \\
\text { vertientes } \\
\text { modificado }\end{array}$ & $\begin{array}{l}\text { Destilador } \\
\text { solar en } \\
\text { terrazas }\end{array}$ \\
\hline \multirow{3}{*}{$\begin{array}{l}\text { Temperatura } \\
\text { media }\end{array}$} & $\mathbf{R}$ & $0,604^{*}$ & 0,503 & $0,708 * *$ & 0,469 \\
\hline & $\begin{array}{l}\text { Sig. } \\
\text { (bilateral) }\end{array}$ & 0,029 & 0,080 & 0,007 & 0,106 \\
\hline & $\mathrm{N}$ & 13 & 13 & 13 & 13 \\
\hline \multirow{3}{*}{$\begin{array}{l}\text { Temperatura } \\
\text { máxima }\end{array}$} & $\mathbf{R}$ & 0,501 & 0,405 & $0,610 *$ & 0,351 \\
\hline & $\begin{array}{l}\text { Sig. } \\
\text { (bilateral) }\end{array}$ & 0,081 & 0,170 & 0,027 & 0,240 \\
\hline & $\mathrm{N}$ & 13 & 13 & 13 & 13 \\
\hline \multirow{3}{*}{$\begin{array}{l}\text { Temperatura } \\
\text { mínima }\end{array}$} & $\mathbf{R}$ & $0,588 *$ & 0,417 & $0,573 *$ & 0,484 \\
\hline & $\begin{array}{l}\text { Sig. } \\
\text { (bilateral) }\end{array}$ & 0,034 & 0,156 & 0,040 & 0,094 \\
\hline & $N$ & 13 & 13 & 13 & 13 \\
\hline \multirow{3}{*}{ Horas de sol } & $\mathbf{R}$ & 143 & 0,056 & $-0,133$ & $-0,254$ \\
\hline & $\begin{array}{l}\text { Sig. } \\
\text { (bilateral) }\end{array}$ & 0,640 & 0,857 & 0,665 & 0,402 \\
\hline & $\mathrm{N}$ & 13 & 13 & 13 & 13 \\
\hline
\end{tabular}

En la figura 4 podemos apreciar que también la producción de agua destilada en cada uno de los módulos tiende a ir incrementándose ligeramente desde el primer día de evaluación del 07 de octubre hasta el 30 del mes de diciembre. 


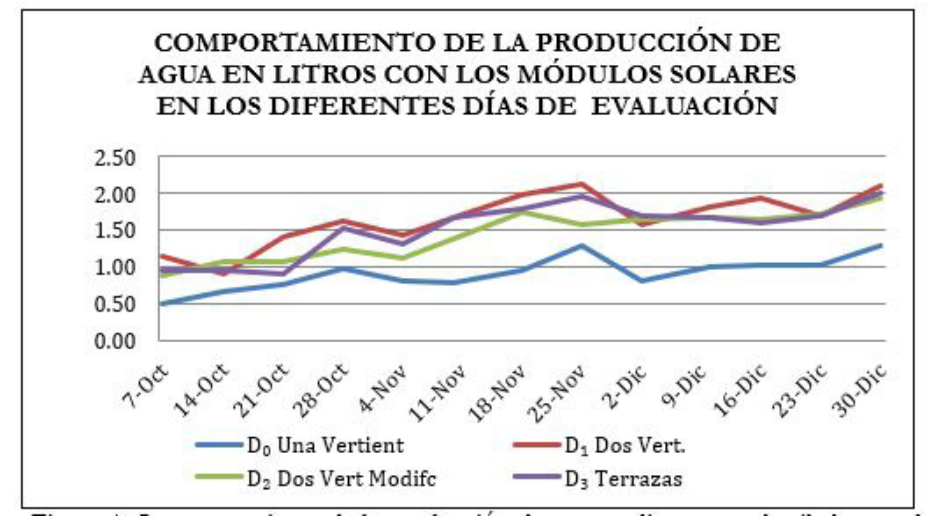

Figura 4. Comportamiento de la producción de agua en litros, con destiladores solares en estudio y en los deferentes días de evaluación

\section{Evaluación de la rentabilidad económica:}

Desde el punto de vista económico y teniendo en cuenta la mayor cantidad de agua lograda con el módulo de dos vertientes de plástico obtenemos la cantidad de 1,65 litros en promedio por día durante los tres meses con un costo más económico de $\mathrm{S} /$ 337,4 el módulo, ahora que si aumentamos la inversión a una cantidad aproximada de S/ 4088,3 esto permitirá producir 20 litros de agua diarios y se puede pagar en un periodo acumulado de 10 años, el costo mensual de agua que correspondería sería de S/ 34 soles (sin considerar intereses) para tener 20 litros diarios de agua destilada como mínimo según la FAO, en el desierto y para consumo humano.

Tabla 4. Rentabilidad económica de los módulos solares en estudio

\begin{tabular}{|c|c|c|c|c|c|c|c|c|}
\hline \multirow[t]{2}{*}{ MODELO DE DESTILADOR } & \multirow[t]{2}{*}{ Descripción } & \multirow{2}{*}{$\begin{array}{l}\text { Costo } \\
\text { Total S/ }\end{array}$} & \multirow{2}{*}{$\begin{array}{c}\text { Cantidad de } \\
\text { agua } \\
\text { destilada } \\
\text { obtenida } \\
\text { promedio de } \\
\text { tres meses } \\
\text { Litros/día }\end{array}$} & $\begin{array}{l}\text { obtenciòn } \\
\text { de } 20 \text { litor } \\
\text { de agua }\end{array}$ & $\begin{array}{c}\text { Costo S/ de agua potable } \\
\text { por mes según EPS Gráu } \\
\left(10 \mathrm{~m}^{3} / \mathrm{mes}\right)\end{array}$ & \multirow{2}{*}{\begin{tabular}{|c} 
Costo S/ de \\
agua potable \\
por mes \\
según EPS \\
Gráu \\
$\left(10 \mathrm{~m}^{3} / \mathrm{mes}\right)$
\end{tabular}} & \multirow{2}{*}{\begin{tabular}{|c|} 
Consto \\
mensual del \\
agua en un \\
horizonte de 10 \\
añospara \\
obtener 20 \\
litros minimos \\
de agua/día en \\
el desierto \\
\end{tabular}} & \multirow{2}{*}{$\begin{array}{l}\text { Costo S/ de } \\
\text { los } 20 \text { litros } \\
\text { de agua dulce } \\
\text { obtenida de } \\
\text { los modulos } \\
\text { según tarifa } \\
\text { de EPS Gráu }\end{array}$} \\
\hline & & & & litros & $\begin{array}{c}\text { Inversión total (S/) para } \\
\text { obtener } 20 \text { litros de agua } \\
\text { por día según cada sistema }\end{array}$ & & & \\
\hline $\begin{array}{l}\text { Destilador solar de una } \\
\text { vertiente (testigo) - } D_{0}\end{array}$ & $\begin{array}{l}\text { Módulo solar con marcos de aluminio, con } \\
\text { paredes de plástico dispuestas en una } \\
\text { vertiente, de } 1 \mathrm{~m}^{2} \text { de base mas cubeta de } \\
\text { metal para contener agua a destilar }\end{array}$ & 430,37 & 0,92 & 20 & 8692,06 & 70 & 72,4 & 0,14 \\
\hline $\begin{array}{l}\text { Destilador solar de dos } \\
\text { vertientes }-D_{1}\end{array}$ & $\begin{array}{l}\text { Modulo solar con marcos de aluminio, con } \\
\text { cubiertas de plastico transparente } \\
\text { dispuestas a dos aguas, } 1 \mathrm{~m}^{2} \text { de base de } \\
\text { vidrio conteniendo a una cubeta de metal. }\end{array}$ & 337,42 & 1,65 & 20 & 4088,34 & 70 & 34,1 & 0,14 \\
\hline $\begin{array}{l}\text { Destilador solar de dos } \\
\text { vertientes modificado }-\mathrm{D}_{2}\end{array}$ & $\begin{array}{l}\text { Modulo solar con marcos de aluminio, con } \\
\text { cubiertas de vidrio transparente dispuestas } \\
\text { a dos aguas y cubiertas o forradas de } \\
\text { plastico transparente, } 1 \mathrm{~m}^{2} \text { de base de vidrio } \\
\text { conteniendo a una cubeta de metal. }\end{array}$ & 571,85 & 1,45 & 20 & 7914,81 & 70 & 66 & 0,14 \\
\hline $\begin{array}{l}\text { Destilador solar de cascada o } \\
\text { en terrazas }-D_{3}\end{array}$ & $\begin{array}{l}\text { Modulo solar en terrazas o cascasda de } \\
\text { vidrio a un agua, } 1 \mathrm{~m}^{23} \text { de base de vidrio }\end{array}$ & 722,18 & 1,53 & 20 & 9468,85 & 70 & 78,9 & 0,14 \\
\hline
\end{tabular}




\section{DISCUSIÓN}

Respecto del promedio de agua destilada obtenida con los cuatro modelos de destilación solar y en los tres meses del experimento encontramos que en el mes de octubre el mayor volumen lo alcanza el destilador solar de dos vertientes (D-1) con 1,279 l/ día promedio siendo significativamente mayor que la producción del destilador solar de una vertiente, pero estadísticamente iguales que los valores obtenidos por los destiladores solares el de dos vertientes modificados (D-2) y el destilador en cascada (D-3). Para el caso de los resultados del mes de noviembre, se observa que la mayor producción de agua destilada lo obtenemos con el modelo de destilador solar de dos vertientes de 1,806 1/día promedio. El que supera significativamente al volumen obtenido del destilador solar de una vertiente que produce 0,960 l/día promedio, y también el destilador solar de dos vertientes tiene una producción estadísticamente similar a la del destilador solar de dos vertientes y a la del destilador escalonado o en terrazas. Para el caso de los resultados obtenidos en el mes de diciembre, podemos decir que el destilador solar de dos vertientes (D-1) nuevamente logra valores significativamente superiores a 1,823 1/ día promedio en comparación con el destilador solar de una vertiente 1,042 1/día promedio, pero tiene una producción estadísticamente similar a la de los destiladores solares de dos vertientes modificados y al destilador solar de cascada.

Desde el punto de vista de producción final a los tres meses del experimento se puede señalar que el destilador de dos vertientes tiene el mayor promedio de producción 1,651 1/día promedio y con un posible rango de amplitud que oscila entre los 1,509 a 1,763 $\mathrm{l}$ /día promedio, siendo diferente y significativamente mayor que el promedio obtenido del destilador solar de una vertiente y estadísticamente similar que los promedios obtenidos de los destiladores solares de dos vertientes modificados y el destilador en cascada con respecto al promedio de volumen de agua obtenido combinando la producción mensual de los cuatro destiladores en estudio obtenidos en cada mes podemos apreciar que el promedio obtenido en el mes de diciembre 1,583 1/día promedio es el mayor significativamente al promedio obtenido en el mes de octubre (1,044 1/día promedio) pero es estadísticamente igual al obtenido en el mes de noviembre. (1,481 1/día promedio)

Respecto a la relación de los factores climáticos y el volumen de agua obtenida con los destiladores podemos afirmar que si bien hay un mayor volumen de agua obtenido del modelo D-1 tanto para una temperatura media y como para una temperatura máxima. También para el modelo D-2 destilador solar a dos aguas modificado y el 
destilador solar testigo D-0 resulta una relación en forma significativa y directa con el comportamiento de la temperatura mínima. A este comportamiento también tendría que evaluarse con mayor detalle la influencia de la altura dada $(1 \mathrm{~m})$ al diseño físico de este módulo solar. También para el modelo de destilador a dos aguas modificado (D-1) se le debe evaluar la influencia de usar dos capas trasparentes una de vidrio y la otra de plástico grueso $\left(\mathrm{N}^{\circ} 16\right)$. A las cubiertas o techos del destilador de dos vertientes se le dio una altura de $50 \mathrm{~cm}$ desde la base del módulo solar.

Respecto a la rentabilidad económica de los módulos solares en estudio podemos afirmar que al evaluar la hipótesis alternativa y la hipótesis nula para ver la posibilidad de producir 20 litros de agua mínima por día, la Hipótesis Alternativa, desde el punto de vista económico y teniendo en cuenta la mayor cantidad de agua lograda con el módulo de dos vertientes de plástico en la que obtenemos la cantidad de 1,65 litros en promedio por día durante los tres meses con un costo de S/ 337,4 el módulo, es viable si aumentamos la inversión a una cantidad aproximada de S/ 4088,3 esto permitirá producir 20 litros de agua diarios y se puede pagar en un periodo acumulado de 10 años, y el costo mensual de agua en la que se incurre sería de S/ 34 soles y sin intereses para tener 20 litros diarios de agua destilada en el desierto y para consumo humano. Cabe señalar que este costo puede disminuirse aún más de la mitad. Dado que las condiciones económicas del ganadero de la zona hacen ver que está en posibilidades de cubrir ese costo y a la vez frecuenta ingresar al desierto una vez a la semana quedándose tres días a la semana en el desierto, es que se ve factible desde ese punto de vista. También podemos decir que el mismo productor no vive los siete días de la semana en el desierto, solo usa dos a tres días, aprovechando para dejar trabajando esos sistemas de destilación de agua y tener la cantidad de agua diaria necesaria y acumulada a la vez.

Respecto a la calidad del agua obtenida, ésta fue sometida a un análisis en los laboratorios de la Universidad de Piura según las normas de la DIGESA. El estudio arrojó que son aguas aptas para el consumo humano. Hay que tener cuidado con usar depósitos limpios al recoger el agua para evitar su posible contaminación. 


\section{CONCLUSIONES}

Los destiladores solares de dos vertientes, el destilador solar de cascada y el destilador solar de dos vertientes modificado con los rendimientos promedios de 1,6510; 1,5250 y 1,4450 litros de agua destilada por $\mathrm{m}^{2} /$ día son iguales estadísticamente y a la vez superan estadísticamente en la producción promedio de agua destilada durante los tres meses al destilador solar de una vertiente 0,921 litros de agua destilada por $\mathrm{m}^{2} /$ día.

Ningún destilador probado logra alcanzar la producción de 20 litros de agua promedio por $\mathrm{m}^{2}$ de espejo de agua y por día. Solo incrementando el número de réplicas para cada modelo en estudio se podría llegar más fácilmente a alcanzar la cantidad de 20 litros de agua destilada por día, mas no por $\mathrm{m}^{2}$ de espejo de agua.

El diseño más adecuado ambiental y económicamente es el destilador de dos vertientes que logra producir mayor volumen de agua: 1,651 litros de agua destilada por $\mathrm{m}^{2} /$ día a un menor costo de S/ 337,32 soles por módulo., y por ser de menor altura usa menor la cantidad de plástico para su diseño.

\section{REFERENCIAS BIBLIOGRÁFICAS}

Erich, Saettone. 2015. Análisis comparativo entre diferentes destiladores solares de agua marina <http://www.perusolar.org/wp-content/uploads/2016/07/Analisis-Comparativo-entre-diferentes-Destiladores-Erich-Saettone.pdf $>$ [Consulta: 17-02-2017].

Fonseca, Susana; Brito, Ángel; Andión, Ronald; Perdomo, Eider; Fernández, María. 2009. Análisis Exergético del Destilador Solar de Bandeja de Fibra de Vidrio. Universidad de Oriente Santiago de Cuba. <http:/ /www.redalyc.org/pdf/4455/445543760003. pdf $>$ [Consulta: 21-08-2018].

McCluney, W. 1984. Solar distillation of Water. Florida Solar Energy Center/University of Central Florida. <http://www.fsec.ucf.edu/en/publications/pdf/FSECEN-3-80pdf $>$ [Consulta: 18-11-2018]. 
| Roberto Chiroque Luján

Ros, Antonio. 2011. El agua. Desalación (4/4). <http://www.mailxmail.com/curso-agua-desalacion-4-4/procesos-desalacion> [Consulta: 14-09-2018].

United Nations Department of Economic and Social Affairs. 1970. Solar Distillation and a Means of Meeting Small-Scale Water Demands. <https://www.archive.org/ stream/fe_solardistilation_for_small-scale_water_demands_djvu.txt $>$ [Consulta: 18-11-2018].

Recepción: 20/11/2018

Aceptación: 20/12/2018

\section{Correspondencia}

Roberto Chiroque Luján

chiroqueroberto@gmail.com 